\title{
«Schielen ist auch ein Stigma»
}

\section{Ronald D. Gerste}

Dr. med., Dr. phil., Facharzt für Augenheilkunde, Medizinhistoriker und Journalist

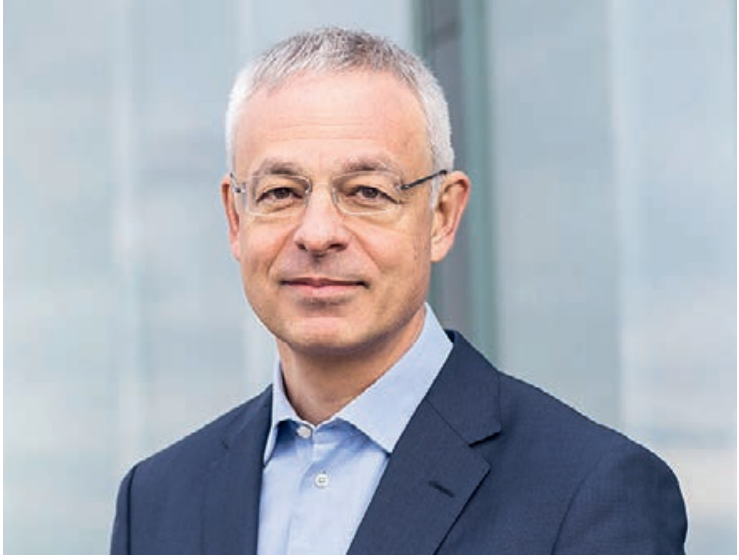

Daniel Mojon

Kinder können grausam sein. Headhunter auch. Beiden Gruppen legte Daniel Mojon Porträtfotos vor. Im Falle der Kinder waren es nicht weniger als 118 junge Patienten und Patientinnen der Augenklinik am Kantonsspital St.Gallen zwischen drei und zwölf Jahren, denen der Ophthalmologe Bilder von sechs Zwillingspaaren zeigte und die er fragte, welche der abgebildeten Kinder sie denn am ehesten zu der eigenen Geburtstagsfeier einladen würden. Der Clou des Ganzen: Mojon hatte einigen der Zwillingskinder per digitale Bildverarbeitung einen Strabismus convergens (das Schielen eines Auges nach innen) oder einen Strabismus divergens (das Schielen nach aussen) verpasst. Nicht ganz überraschend für Mojon: Schielende Kinder wurden deutlich seltener zu den (hypothetischen) Geburtstagsfeiern eingeladen. Immerhin: Die Jüngeren im Kollektiv; jene, die sechs Jahre und weniger alt waren, diskriminierten nicht und luden Schieler und Orthophore (Menschen mit Parallelstand der Augen) in gleichem Masse ein. Nicht viel anders verlief Mojons Studie mit Headhuntern. Auch hier arbeitete der Augenarzt mit Porträts, die er per Photoshop verändert hatte. Unter den dabei den Gesichtern zugefügten kosmetischen Besonderheiten war ein Schielen ein stärkeres Hindernis, einen Job zu bekommen, als eine digital der Physiognomie zugefügte Akne oder ein ähnlich virtueller fehlender Schneidezahn.

«Schielen ist nicht nur eine Gefährdung in der Entwicklung des visuellen Systems im Kleinkindesalter", betont Daniel Mojon, «die frühestmöglich behandelt werden muss, um einer Amblyopie vorzubeugen. Der
Strabismus ist auch ein Stigma. Schieler werden wenn das Wortspiel erlaubt ist - von ihren Mitmenschen schief angesehen, erleiden mannigfache Nachteile. Deshalb ist die Strabismuschirurgie nicht nur eine funktionelle, nicht nur eine kosmetische Intervention, sondern ein entscheidender Beitrag zu gesunder sozialer Befindlichkeit und zur Lebensqualität.»

\section{Seltene Ehrung}

Die Strabismuschirurgie, die Intervention an einem oder mehreren der sechs Augenmuskeln, ist durch Mojon in den letzten Jahren um eine besondere Option bereichert worden. Der Ophthalmologe führte die im internationalen Schrifttum inzwischen fast durchgängig als MISS (Minimally Invasive Strabismus Surgery) bezeichnete neue Technologie ein, die mit wesentlich kleineren Inzisionen als die herkömmliche Operationsmethode auskommt und eine schnellere Wundheilung sowie visuelle Rehabilitation ermöglicht. Der Pionier erfuhr vor kurzem eine nicht alltägliche Ehrung: Als erster Schweizer Augenarzt war er eingeladen, die Keynote Lecture auf der Jahrestagung der Deutschen Ophthalmologischen Gesellschaft (DOG) in Bonn zu halten - die DOG, deren Gründung auf den Urvater der modernen Augenheilkunde, Albrecht von Graefe, in den 1850er Jahren zurückgeht und als die älteste medizinische Fachgesellschaft der Welt gilt, erteilt eine solche Würdigung nur in sehr bescheidenem Mass.

Dass die Eröffnung der Bindehaut in der klassischen Schielchirurgie als "türflügelartig» bezeichnet wird, spricht Bände. Bei der von Mojon für verschiedene Interventionen konzipierten und weiterentwickelten MISS hingegen werden statt einer grossen Bindehauteröffnung mehrere kleine - unter dem Operationsmikroskop - angelegt, und dies fern des Limbus auf eine Weise, dass die selbstschliessenden Inzisionen postoperativ von den Lidern bedeckt sind. Die Narbenbildung ist bei diesem Vorgehen minimal und die Operationszeit (in den Händen eines erfahrenen Chirurgen oder einer erfahrenen Chirurgin) sehr kurz. Inzwischen ist eine ganze Reihe von Eingriffen erfolgreich auf diese minimal-invasive Weise erfolgreich vorgenommen worden wie Rücklagerungen, Faltungen, Resektionen und Transpositionen der geraden (recti) und der schrägen Augenmuskeln (obliqui). 

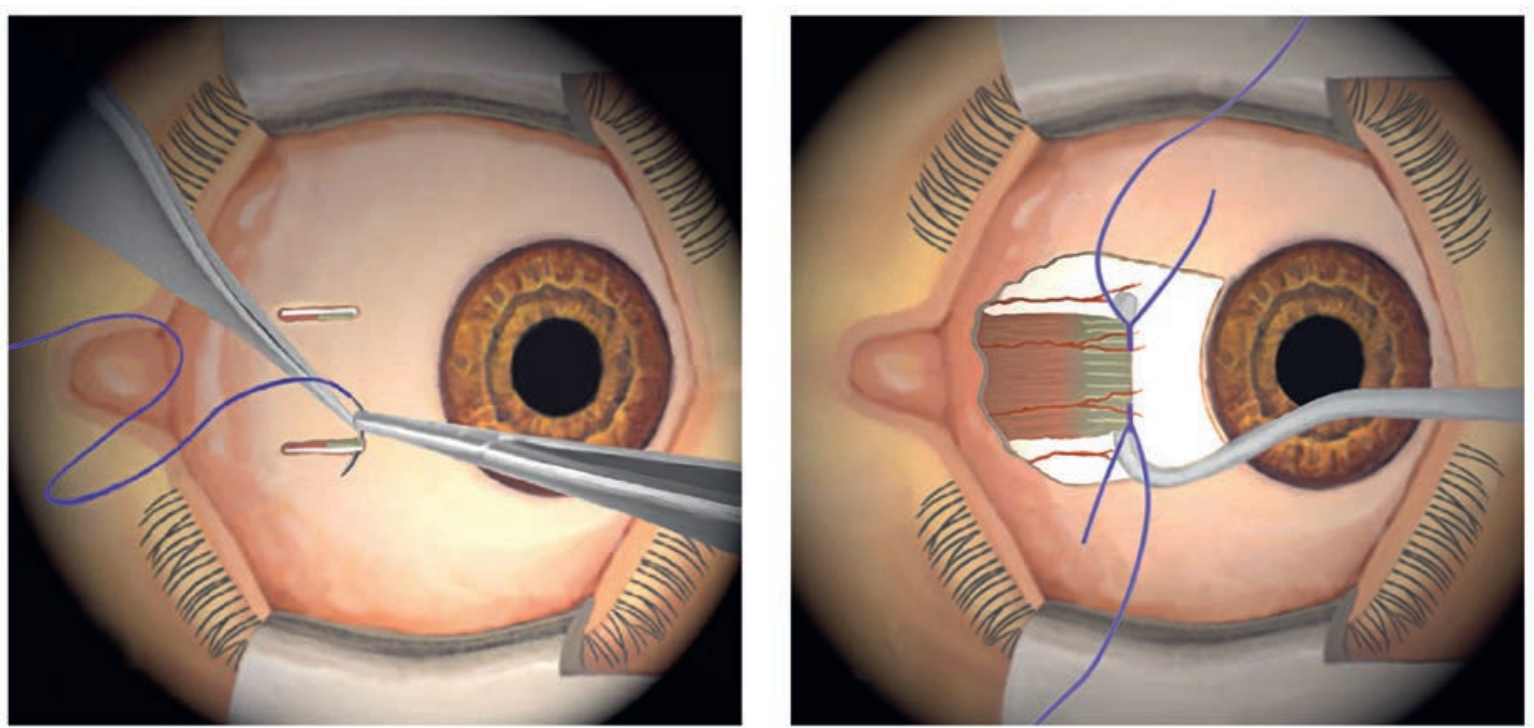

Schieloperationstechniken im Vergleich:

Links die MISS mit der kaum sichtbaren Inzision, rechts die klassische Methode mit grosszügig eröffneter Bindehaut.

\section{Der Augenchirurg als Künstler}

Mojon konnte das Publikum bei seinem Auftritt in Bonn nicht nur von der MISS begeistern, sondern gab auch einen Eindruck von seiner Vielseitigkeit: Der Augenchirurg sucht nach Wegen, auch andere Interventionen jenseits der Schielbehandlung weniger invasiv zu machen - wobei Invasivität in diesem Fach angesichts von Inzisionen in der Grössenordnung weniger Millimeter ein relativer Begriff ist. $\mathrm{Zu}$ seinen jüngsten Erfindungen gehört eine neue Glaukomoperation, die ebenfalls im Vergleich zu einer etablierten Methode das Ziel der Senkung des Augeninnendrucks weniger traumatisch erreichen soll.

Die diffizile Chirurgie am Auge ist für Daniel Mojon der feinfühligen Arbeit eines Künstlers vergleichbar. Die Neigung zu dieser Muse liegt ihm im Blut: Der Vater des 1963 in Bern geborenen Ophthalmologen war der Kunsthistoriker Luc Mojon. Daniel studierte Humanmedizin in Bern und an der Columbia University in New York. In Bern leitete er die Abteilung für Strabologie und Neuroophthalmologie und die Glaukomsprechstunde an der Universitätsaugenklinik, in St.Gallen wurde er Leitender Arzt der Augenklinik. Heute vereint er das ärztliche Wirken mit dem eines Wissenschafters (von beträchtlichem publizistischem Output) an drei Standorten: Mojon praktiziert und operiert in Praxiskliniken in St.Gallen, am Airport Zürich, in Winterthur, Zürich und in Heiden - Letzteres mehr als eine geographische Fussnote in der Geschichte der Augenheilkunde: Der bereits erwähnte Albrecht von Graefe pflegte hier wiederholt zur Erholung zu verweilen und erhoffte sich Linderung seiner Tuberkulose, indes vergeblich. Mojon ist mit der Gesundheitsökonomin Stefania Mojon-Azzi verheiratet, das Paar hat 3 Kinder; ein Sohn studiert in Zürich Medizin, ein Sohn absolviert in Paris seinen Master in Physik, eine Tochter besucht die Primarschule.

\section{Nie mit dem Erreichten zufrieden}

"Als Ophthalmochirurg», so erklärt Mojon nachdenklich, «steht man immer vor der Herausforderung, nie mit dem Erreichten zufrieden zu sein und an weiteren Verbesserungen der Eingriffe zu arbeiten. Das ganz besondere an der Strabismuschirurgie ist, dass es für erfolgreiche Eingriffe ein dreidimensionales Vorstellungsvermögen braucht.»

Die extreme Feinheit, deren es bei einer MISS bedarf, das Anschlingen eines nur wenige Millimeter dicken Muskels mit einem Faden, seine Rücklagerung ebenfalls auf den Dezimal-Millimeter berechnet, das Agieren unter dem Operationsmikroskop durch eine schlüssellochartige Öffnung sind Facetten einer Chirurgie, die Lichtjahre entfernt scheinen von der Tätigkeit eines Orthopäden oder eines Viszeralchirurgen. Das Entfernungsmass ist das Stichwort: Derart im Kleinen, fast im Submikroskopischen arbeitend, sucht der wissenschaftsbegeisterte Mojon in seiner Freizeit Erkenntnis im Grösstmöglichen, dem Universum: Mit einem Instrumentarium, das kaum noch das Präfix «Amateur-» rechtfertigt, widmet er sich in klaren Bergnächten der Astronomie.

\section{Bildnachweis}

Foto Daniel Mojon zur Verfügung gestellt vom Fotografierten, (c) Raimo Rudi Rumpler

Abbildungen Augenoperationstechniken: Zeichnungen

von Daniel Mojon, von diesem zur Verfügung gestellt. 